\title{
Grand challenges for archaeology
}

\section{Keith W. Kintigh ${ }^{a, 1}$, Jeffrey H. Altschul ${ }^{b}$, Mary C. Beaudry', Robert D. Drennan ${ }^{d}$, Ann P. Kinzige, Timothy A. Kohler ${ }^{\mathrm{f}, \mathrm{g}}$, W. Fredrick Limp' ${ }^{h}$, Herbert D. G. Maschneri, William K. Michener', Timothy R. Pauketatk ${ }^{\text {, }}$ Peter Peregrine ${ }^{g, 1}$, Jeremy A. Sabloff', Tony J. Wilkinson ${ }^{m}$, Henry T. Wright ${ }^{\text {g,n }}$, and Melinda A. Zeder ${ }^{\circ}$}

aschool of Human Evolution and Social Change, Arizona State University, Tempe, AZ 85287; bStatistical Research, Inc., Tucson, AZ 85751; ' Department of Archaeology, Boston University, Boston, MA 02215; 'Center for Comparative Archaeology, Department of Anthropology, University of Pittsburgh, Pittsburgh, PA 15260; ' School of Life Sciences, Arizona State University, Tempe, AZ 85287; ' Department of Anthropology, Washington State University,

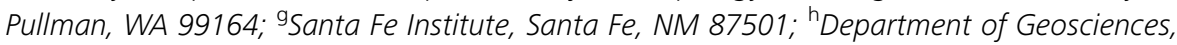
University of Arkansas, Fayetteville, AR 72701; 'Idaho Museum of Natural History, Idaho State University, Pocatello, ID 83209; 'University Libraries, The University of New Mexico, Albuquerque, NM 87131; ' Department of Anthropology, University of Illinois at UrbanaChampaign, Urbana, IL 61801; 'Department of Anthropology, Lawrence University, Appleton, WI 54911; ' 'Department of Archaeology, Durham University, Durham DH1 3LE, United Kingdom; " Museum of Anthropology, Ann Arbor, MI 48109; and ${ }^{\circ}$ Department of Anthropology, National Museum of Natural History, Smithsonian Institution, Washington, DC 20013

Archaeology is a source of essential data regarding the fundamental nature of human societies. Researchers across the behavioral and social sciences use archeological data in framing foundational arguments. Archaeological evidence frequently undergirds debate on contemporary issues. We propose here to answer "What are archaeology's most important scientific challenges?" The question arose as we sought to develop recommendations for investments in computational infrastructure that would enable the discipline to address its most compelling questions. Absent a list of these questions, we undertook to develop our own.

The project steering committee was composed of archaeologists (K.W.K., J.H.A., W.F.L., and J.A.S.), an ecologist (A.P.K.), and an information scientist (W.K.M.). The steering committee selected other participants (the additional authors), striving to compose a small group of experts whose work broadly represents areas of the world, diverse theoretical perspectives, and the range of social complexity in the past.

A spring 2012 crowd-sourcing effort was effected through e-mail requests and listserv postings by the major North American and European professional associations. We asked our colleagues to identify problems of broad scientific and social interest that could drive cutting edge research in archaeology for the next decade and beyond. We received
181 responses to the Web-based survey (1) that requested a concise statement of a grand challenge problem and, optionally, a justification of its importance and demographic information about the respondent. The self-selected respondents were primarily from the United States and were split across academic, consulting, and government employment sectors.

The authors then convened in a summer 2012 workshop at the Santa Fe Institute to augment, prioritize, and refine the crowdsourced grand challenges. It was agreed that the problems should have global significance, although they may address processes operating at spatial scales from households to empires and at a broad range of temporal scales. The challenges had to be, in principle, susceptible to a solution supported by data Before viewing the crowd-sourced suggestions, we proposed and prioritized our own challenges. Then we considered and culled the crowd-sourced challenges that described fundamental problems in science and integrated them with those we had developed independently, resulting in the 25 grand challenges presented in Box 1. After the workshop, the authors wrote concise summaries with illustrative references for each challenge (2).

These challenges focus on understanding the dynamics of cultural processes and the operation of coupled human and natural systems, recognizing that humans-mediated by culture-both affect and are affected by their natural environments. The challenges addressed questions of emergence, complexity, demography, mobility, identity, resilience, and human-environment interactions. There is a notable lack of concern with the earliest, the largest, and the otherwise unique. They show an increasing concern with relevance to the contemporary world. There is no lack of regard for prehistory; the facts of the past provide the evidence that is essential to confront all of these questions. We harbor no illusions about the difficulties of addressing these classes of problems. Rather, we share a conviction that these are the domains in which the most important problems reside.

Many of the cultural processes implicated here undoubtedly involve complex, nonlinear relationships in which cause and effect are not readily distinguished. Further complicating our task, short-term human responses to problems often have unintended consequences, in both the short and long terms. As a consequence, addressing many of these challenges will require both sophisticated modeling and large-scale synthetic research that are only now becoming possible.

Although new archaeological field work will be needed, the greatest payoff will derive from exploiting the explosion in systematically collected archaeological data that has occurred since the mid-20th century, largely in response to laws protecting archaeological resources. Both the needed modeling and synthetic research will require far more comprehensive online access to thoroughly documented research data and to unpublished reports detailing the contextual information essential for the comparative analyses. Indeed, our survey emphatically reinforced the need for the kinds of online access provided by the Digital Archaeological Record (United States) and the Archaeology Data Service (United Kingdom). Although the challenges offered here situate archaeology squarely within the landscape of contemporary science,

Author contributions: K.W.K., J.H.A., M.C.B., R.D.D., A.P.K., T.A.K., W.F.L., H.D.G.M., W.K.M., T.R.P., P.P., J.A.S., T.J.W., H.T.W., and M.A.Z. wrote the paper.

${ }^{1}$ To whom correspondence should be addressed. E-mail: kintigh@ asu.edu.

Any opinions, findings, conclusions, or recommendations expressed in this work are those of the author and do not necessarily reflect the views of the National Academy of Sciences. 


\section{Box 1. Grand challenges for archaeology}

A. Emergence, communities, and complexity

1. How do leaders emerge, maintain themselves, and transform society?

2. Why and how do social inequalities emerge, grow, persist, and diminish, and with what consequences?

3. Why do market systems emerge, persist, evolve and, on occasion, fail?

4. How does the organization of human communities at varying scales emerge from and constrain the actions of their members?

5. How and why do small-scale human communities grow into spatially and demographically larger and politically more complex entities?

6. How can systematic investigations of prehistoric and historic urban landscapes shed new light on the social and demographic processes that drive urbanism and its consequences?

7. What is the role of conflict-both internal factional violence and external warfare-in the evolution of complex cultural formations?

B. Resilience, persistence, transformation, and collapse

1. What factors have allowed for differential persistence of societies?

2. What are the roles of social and environmental diversity and complexity in creating resilience and how do their impacts vary by social scale?

3. Can we characterize social collapse or decline in a way that is applicable across cultures, and are there any warning signals that collapse or severe decline is near?

4. How does ideology structure economic, political, and ritual systems?

C. Movement, mobility, and migration

1. What processes led to, and resulted from, the global dispersal of modern humans?

2. What are the relationships among environment, population dynamics, settlement structure, and human mobility?

3. How do humans occupy extreme environments, and what cultural and biological adaptations emerged as a result?

4. Why does migration occur and why do migrant groups maintain identities in some circumstances and adopt new ones in others?

D. Cognition, behavior, and identity

1. What are the biophysical, sociocultural, and environmental interactions out of which modern human behavior emerged?

2. How do people form identities, and what are the aggregate long-term and large-scale effects of these processes?

3. How do spatial and material reconfigurations of landscapes and experiential fields affect societal development?

E. Human-environment interactions

1. How have human activities shaped Earth's biological and physical systems, and when did humans become dominant drivers of these systems?

2. What factors drive or constrain population growth in prehistory and history?

3. What factors drive health and well-being in prehistory and history?

4. Why do foragers engage in plant and animal management, and under what circumstances does management of a plant or animal lead to its domestication?

5. Why do agricultural economies emerge, spread, and intensify, and what are the relationships among productive capacity, population, and innovation?

6 . How do humans respond to abrupt environmental change?

7. How do humans perceive and react to changes in climate and the natural environment over short- and long-terms? addressing them will require demanding, long-term cross-disciplinary collaborations that have the potential to yield transformative results with impacts cascading far beyond archaeology.

ACKNOWLEDGMENTS. We thank the anonymous archaeologists who proposed challenges through the Web-based survey and the Santa Fe Institute for hosting the grand challenge workshop. This work was supported by National Science Foundation Grant 1202413.

1 Kintigh KW (2013) Grand challenges for archaeology: Crowd sourcing report. Available at https://core.tdar.org/document/391233. Accessed January 6, 2014

2 Kintigh KW, et al. (2014) Grand challenges for archaeology. Am Antiq 79(1):5-24 